\title{
Role of microRNAs in skeletal muscle development and rhabdomyosarcoma (Review)
}

\author{
HUIMING JU, YUEFEI YANG, ANZHI SHENG and XING JIANG \\ College of Veterinary Medicine, Yangzhou University, Yangzhou, Jiangsu 225009, P.R. China
}

Received May 11, 2014; Accepted January 15, 2015

DOI: $10.3892 / \mathrm{mmr} .2015 .3275$

\begin{abstract}
Skeletal muscle accounts for $\sim 40 \%$ of total body mass. The principle functions of skeletal muscle include supporting the body structure, controlling motor movements and storing energy. Rhabdomyosarcoma (RMS) is a skeletal muscle-derived soft tissue tumor widely occurring in the pediatric population. In previous years, microRNAs (miRNAs) have been demonstrated to be important in skeletal muscle development, function and the pathogenesis of various diseases, including RMS. The present review provided an overview of current knowledge on the muscle-specific and ubiquitously-expressed miRNAs involved in skeletal muscle differentiation and their dysregulation in RMS. Additionally, the potential use and challenges of miRNAs as therapeutic targets in this soft-tissue sarcoma were examined and the future prospects for miRNAs in muscle biology and muscle disorders were discussed.
\end{abstract}

\section{Contents}

1. Introduction

2. Process of skeletal muscle development

3. Expression patterns of miRNAs in skeletal muscle development

4. Muscle-specific miRNAs in myogenesis

5. Non-muscle-specific miRNAs in myogenesis

6. Muscle-specific miRNAs in rhabdomyosarcoma

7. Non-muscle-specific miRNAs in rhabdomyosarcoma

8. miRNAs as novel therapeutic targets in rhabdomyosarcoma

9. Conclusion

Correspondence to: Professor Huiming Ju, College of Veterinary Medicine, Yangzhou University, 12 Wenhui East Road, Yangzhou, Jiangsu 225009, P.R. China

E-mail: huimingju1@126.com

Key words: skeletal muscle, rhabdomyosarcoma, microRNA, therapeutic targets

\section{Introduction}

Muscle is an important part of the animal body and skeletal muscle constitutes $\sim 40 \%$ of total body weight. The principal functions of skeletal muscle include maintaining body structure and posture, controlling motor movement and storing energy (1). Rhabdomyosarcoma (RMS) is a skeletal muscle-derived sarcoma occurring predominantly in children and young adults (2). There are two main subtypes of RMS: Embryonal RMS (eRMS) and alveolar RMS (aRMS). eRMS occurs more often in children $<10$ years old, whereas aRMS prototypically occurs in adolescents in 30\% of RMS cases with a poorer prognosis and a higher rate of metastasis (3). In addition, aRMS exhibits typical chromosomal translocations between chromosomes 2 and 13 [ $\mathrm{t}(2 ; 13)(\mathrm{q} 35 ; \mathrm{q} 14)]$ or chromosomes 1 and 13 [ $t(1 ; 13)$ (q36;q14)], which lead to the production of two fusion genes: paired box (PAX)3/forkhead box protein O1 (FOXO1) and PAX7/FOXO1, respectively (4). Furthermore, although RMS tumors commonly form from within skeletal muscle, they can also originate from non-muscle sites, including the skull base, genitourinary tract, biliary tree and salivary glands $(5,6)$.

Previously, microRNAs (miRNAs), a novel class of small non-coding RNAs, have been demonstrated to act as key regulators of skeletal muscle cell fate determination and to be dysregulated in aRMS and eRMS (7). miRNAs are single-strand RNAs of 22 nucleotides in length, which negatively regulate gene expression at the post-transcriptional level by complementary binding to the $3^{\prime}$ untranslated regions of target genes and result in mRNA degradation or translation inhibition (8). To date, emerging evidence has demonstrated that miRNAs are critical in a considerable number of physiological and pathological processes, including proliferation, differentiation, chemoresistance and tumorigenesis (9-12). It was also reported that overexpression of selected 'tumor suppressor' miRNAs by gain-of function studies impaired the tumorigenic behavior of RMS cells (13). In addition, miRNA expression profiling has been demonstrated to be a promising approach to discriminate specific variants among RMS subtypes and further provide useful prognostic information concerning the alveolar and embryonal forms of RMS $(14,15)$. These studies suggest that miRNA dysregulation may be involved in the pathogenesis of RMS.

The present review aimed to evaluate our current understanding of the regulation of miRNAs in skeletal muscle 
development and their deregulation in RMS. Additionally, the possible therapeutic application and challenges of miRNAs in clinical practice were discussed.

\section{Process of skeletal muscle development}

The skeletal muscle system of vertebrates originates through a complex and multi-stage process termed myogenesis where numerous genes are co-operatively involved in the regulation of each stage (Fig. 1). This process begins in the somites of the embryo, which differentiate into dermomyotome-containing myogenic precursors at the first stage. Following commitment to a myogenic cell lineage, the myogenic precursors proliferate and differentiate into myoblasts, followed by differentiation into myotubes and finally differentiate into myofibers (16).

The regulatory network leading to the process of muscle development has been ascribed to a specific class of transcription factors termed myogenic regulatory factors (MRFs) (17). The expression of MRFs is limited to the muscle lineage and results in the activation of a cascade of events leading to the formation of mature muscle fibers.

The upstream regulators of early MRFs are paired-domain- and homeobox-containing proteins, including Pax3 and Pax7, which are active in embryogenesis. As myoblasts migrate, myogenin (MyoG) and MRF4 are expressed and trigger myoblasts to differentiate into myotubes. Following the terminal differentiation of myotubes, they act in concert with other factors, including myocyte enhancer factor 2 (MEF2) and serum response factor (SRF) to activate genes responsible for muscle fiber architecture and functionality (18). Besides these intrinsic signaling pathways, the differentiating muscle cells are regulated by external stimuli, including transforming growth factor (TGF) $-\beta$ or Wnt signaling (19). In addition, the majority of the aforementioned processes can also be modulated at the post-transcriptional level by miRNAs, which are demonstrated to be irreplaceable in skeletal muscle development.

\section{Expression patterns of miRNAs in skeletal muscle de- velopment}

During skeletal muscle development, certain miRNAs are specifically enriched in skeletal muscle cells and others are differentially expressed in the development process. The temporal or tissue specific expression patterns of miRNAs have been determined by miRNA array or high-throughput sequencing approaches in previous years(20). In one study, 77 miRNAs were found to be upregulated and 68 miRNAs were downregulated by microarray in $\mathrm{C} 2 \mathrm{C} 12$ myoblast cells, which were induced to differentiate in horse serum (20). Among the 77 upregulated miRNAs, miR-133a-1, miR-133a-2, miR-133b and miR-206 were the most significantly upregulated. Their critical role in skeletal muscle differentiation was also confirmed by other studies (21-24). Several other miRNAs, including miR-9-2, miR-122a, miR-703 and miR-805, were most significantly downregulated, however, few of them were found to be involved in the differentiation process. Certain miRNAs, including miR-699a, were downregulated during skeletal muscle differentiation. These observations demonstrated that miRNAs were differentially expressed in the process of skeletal muscle development.

\section{Muscle-specific miRNAs in myogenesis}

Numerous miRNAs can be highly and specifically enriched in certain tissues. The miRNAs that are specifically expressed in skeletal muscle are referred to as myomiRs, which include the miR-1/206 cluster $(21,25)$. A complete list of myomiRs is provided in Table I and their function is further described in corresponding paragraphs. The miR-1/206 cluster is composed of six distinct miRNAs located on three separate chromosomes in three bicistronic transcripts. miR-1-2 and miR-133a-1 are located on chromosome 18, miR-1-1 and miR-133a-2 are located on chromosome 20 and miR-133b along with miR-206 are located on chromosome 6 (Fig. 2). In terms of architecture, miR-1-1 and miR-1-2 are identical and differ from miR-206 by four nucleotides. miR-133a-1 and miR-133a-2 are identical and differ from miR-133b by one nucleotide. During myogenesis, the myogenic transcription factors MyoD, MEF2 and SRF directly regulate the expression of miR-1 and miR-133a in skeletal muscle whereas the expression of miR-206 is controlled by MyoD and MyoG $(26,27)$.

The function of the muscle-specific miRNAs in myogenesis has been examined in detail. It was reported that miR-1 and miR-133a regulate skeletal muscle cell proliferation and differentiation by targeting histone deacetylase 4 (HDAC4) and SRF, respectively, thus establishing a negative-feedback loop for myocyte differentiation (21). Furthermore, the injection of miR-1 into embryonic cardiomyocytes of mice led to decreased proliferation of cardiomyocytes, which was ascribed to the decreased expression of Hand2, a transcription factor that promotes cardiomyocyte proliferation (26). Consistently, a significant increase in Hand2 expression and proliferating cardiomyocytes was observed in an miR-1-2 deficient mouse model (28).

By contrast, miR-133a promotes myoblast proliferation partly by repressing the expression of SRF, a critical regulator of muscle cell differentiation (21). The genetic interaction between miR-133a and SRF results in the upregulation of miR-133a by SRF leading to the further repression of SRF, thereby constituting a negative feedback loop. Paradoxically, miR-133a and miR-1 exhibit opposing effects on skeletal muscle development although they derive from the same miRNA polycistronic transcript. The primary function of miR-133a is to promote proliferation and inhibit differentiation, while the function of miR-1 is to induce the differentiation of mesodermal progenitors to the muscle lineages. It has been demonstrated that miR-1 and miR-133 have a specific role in muscle cell proliferation and differentiation in an antagonistic manner, with the balance being altered one way or the other by additional modulators of gene expression (29).

Similar to miR-1 in skeletal muscle, miR-206 has been demonstrated to promote myoblast differentiation by repressing the expression of connexin 43 (Cx43), thereby decreasing the electrical coupling between myofibers via gap junctions, which inhibits the terminal differentiation of skeletal muscle cells (30). In addition, miR-206 was reported to repress the expression of the p180 subunit of DNA polymerase $\alpha 1$ (22), Pax7 (31), follistatin-like 1 (32) or utrophin (32), thereby suppressing muscle cell proliferation through inhibiting DNA synthesis. 
Table I. Muscle-specific miRNAs involved in myogenesis and RMS.

\begin{tabular}{|c|c|c|c|}
\hline miRNA & Target gene & Function & Reference \\
\hline \multirow[t]{6}{*}{ miR-1 } & HDAC4 & Promotes myoblast differentiation & 20 \\
\hline & Hand2 & Inhibits cardiomyocyte proliferation & 25,27 \\
\hline & PAX3 & Inhibits RMS cell proliferation & 41 \\
\hline & PAX7 & Promotes muscle cell differentiation & 29 \\
\hline & CCND2 & Inhibits RMS cell proliferation & 41 \\
\hline & cMet & Inhibits RMS development & 40 \\
\hline $\operatorname{miR}-133$ & SRF & Promotes myoblast proliferation & 20 \\
\hline \multirow[t]{8}{*}{ miR-206 } & PAX7 & Inhibits muscle cell proliferation & 29 \\
\hline & PAX3 & Inhibits RMS cell proliferation & 41 \\
\hline & CCND2 & Inhibits RMS cell proliferation & 41 \\
\hline & cMet & Inhibits RMS development & 40 \\
\hline & $\mathrm{Cx} 43$ & Promotes myoblast differentiation & 28 \\
\hline & Pol $\alpha 1$ & Inhibits muscle cell proliferation & 21 \\
\hline & Fstl1 & Inhibits muscle cell proliferation & 30 \\
\hline & Utrn & Inhibits muscle cell proliferation & 30 \\
\hline
\end{tabular}

miRNA, microRNA; RMS, rhabdomyosarcoma; HDAC4, histone deacetylase 4; PAX, paired box; SRF, serum response factor; Cx43, connexin 43; Pol $\alpha$, DNA polymerase $\alpha$; Fstl1, follistatin-like 1; Utrn, utrophin.

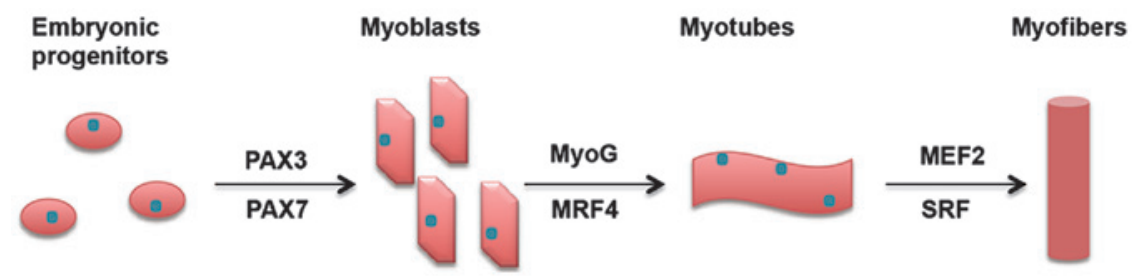

Figure 1. Schematic illustration of skeletal muscle development. During myogenesis, PAX3 and PAX7 are initially activated in embryonic progenitors. With the continuous development of embryos, certain embryonic progenitor cells differentiate into muscle precursor cells termed myoblasts. As myoblasts migrate, MyoG and MRF4 are expressed, which stimulate myoblasts to differentiate into myotubes. They then act with MEF2 and SRF to activate genes responsible for muscle fiber (myofibers) architecture and functionality. PAX, paired box; MyoG, myogenin; MRF4, myogenic regulatory factor 4; MEF2, myocyte enhancer factor-2; SRF, serum response factor.

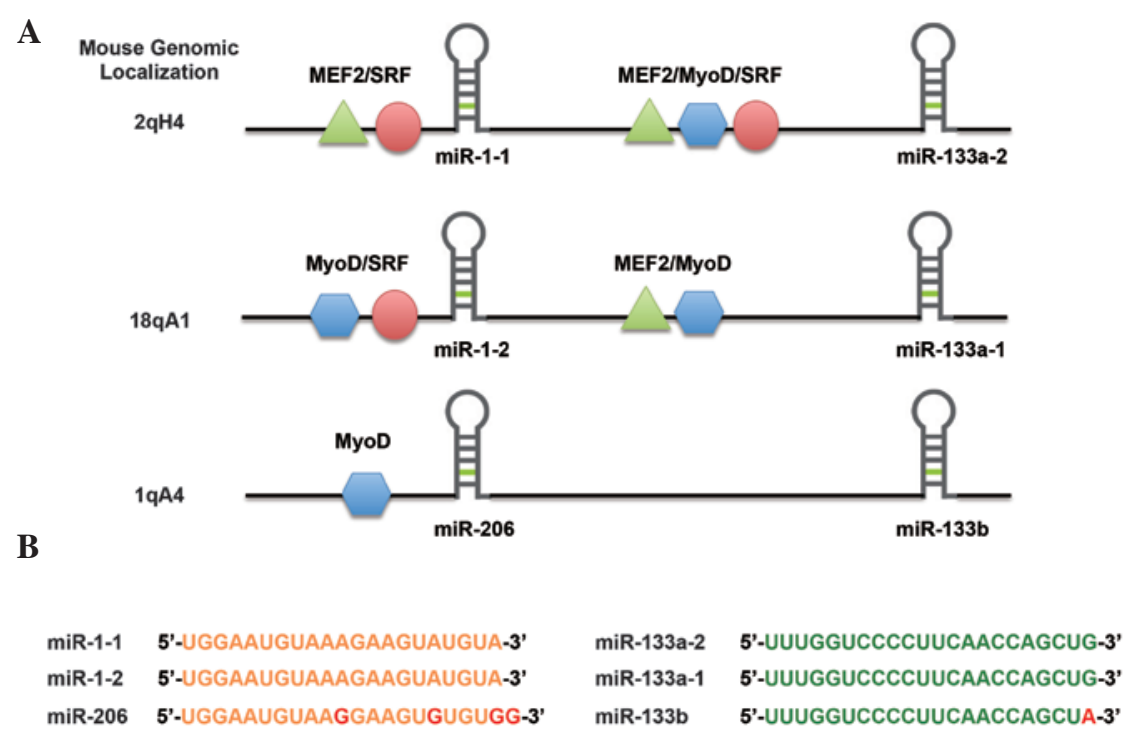

Figure 2. Schematic overview of three myomiR clusters. (A) Genomic locations of the three bicistronic myomiR clusters, including miR-1-1/miR-133a-2, miR-1-2/miR-133a-1 and miR-206/miR-133b on mouse chromosomes. The myogenic regulatory factors SRF, MEF2 and MyoD are also indicated. (B) Comparison of the sequences of three bicistronic myomiR clusters (5'-3'). Red indicates the different nucleotides among the miRNA families. miR, microRNA; MEF2, myocyte enhancer factor-2; SRF, serum response factor. 
Table II. Non-muscle-specific miRNAs involved in myogenesis and RMS.

\begin{tabular}{|c|c|c|c|}
\hline miRNA & Target gene & Function & Reference \\
\hline $\operatorname{miR}-27 b$ & PAX3 & Promotes myoblast differentiation & 31 \\
\hline miR-26a & Ezh2 & Promotes myoblast differentiation & 32 \\
\hline miR-214 & Ezh2 & Promotes myoblast differentiation & 33,34 \\
\hline $\operatorname{miR}-181$ & HOX11 & Promotes muscle cell differentiation & 35 \\
\hline miR-669a & MyoD & Inhibits skeletal muscle differentiation & 36 \\
\hline \multicolumn{4}{|l|}{$\operatorname{miR}-669 q$} \\
\hline \multirow[t]{3}{*}{$\operatorname{miR}-29$} & YY1 & Promotes myoblast differentiation & 42 \\
\hline & PAX3 & Inhibits RMS cell proliferation & 41 \\
\hline & CCND2 & Inhibits RMS cell proliferation & 41 \\
\hline \multirow{2}{*}{ miR-183 } & PTEN & Promotes RMS cell migration & 43 \\
\hline & EGR1 & Promotes RMS cell migration & 43 \\
\hline miR-203 & P63 & Inhibits RMS cell proliferation & 44 \\
\hline $\operatorname{miR}-9^{a}$ & E-cadherin & Inhibits RMS cell migration & 45 \\
\hline $\mathrm{miR}-450 \mathrm{~b}$ & TGF- $\beta 1$ & Inhibits RMS development & 46 \\
\hline
\end{tabular}

miRNA, microRNA; RMS, rhabdomyosarcoma; PAX, paired box; Ezh2, enhancer of zeste 2; HOX11, homeobox-protein A11; YY1, Yin Yang 1; PTEN, phosphatase and tensin homolog; EGR1, early growth response 1 ; TGF- $\beta 1$, transforming growth factor- $\beta 1$.

\section{Non-muscle-specific miRNAs in myogenesis}

In addition to muscle-specific miRNAs, numerous non-muscle-specific miRNAs, referred to as non-myomiRs, are also important in the regulation of myogenesis (Table II). It has been demonstrated that these non-myomiRs regulate muscle proliferation and differentiation through the repression of target genes through multiple processes (33-39). At the onset of myogenesis, miR-27b induced improper migration and early differentiation of myoblasts by targeting the Pax3 protein (33). miR-26a (34) and miR-214 $(35,36)$ also promote myogenesis by targeting enhancer of zeste 2 , another known inhibitor of myogenesis. It is noted that the timing of expression of miR-26a and miR-214 differs during myogenesis. Once muscle differentiation begins, miR-214 is upregulated via MyoD/MyoG, which promote P21 $1^{\mathrm{Cip} 1}$ and myogenin expression, while miR-26a increases gradually during the course of myogenesis.

Inhibition of homeobox-protein A11 (HOX11) by miR-181 is another step in muscle differentiation (37). The low expression of HOX11 leads to increases in MyoD, a target of HOX11, and proper differentiation in muscle cells. It is consistent with the finding that miR-181 is upregulated during muscle development whereas it is downregulated in adult skeletal muscle (38). By contrast, miR-669a and miR-669q are expressed in the heart muscle to prevent skeletal muscle differentiation from the beginning through inhibiting $\mathrm{MyoD}$ and its targets, thus ensuring that skeletal muscle myogenesis occurs in the correct locations (39).

Taken together, these observations are consistent with differential expression profiles of miRNAs between myogenesis and the adult skeletal muscle. The differentially expressed miRNAs provide a molecular basis for proper regulation of muscle development, which highlights the complexity of miRNA function.

\section{Muscle-specific miRNAs in rhabdomyosarcoma}

RMS is predominantly a pediatric sarcoma that resembles developing skeletal muscle and accounts for $>50 \%$ of soft tissues sarcomas in children (40). Evidence has demonstrated that myomiRs were significantly downregulated in RMS, indicating a critical role in the terminal differentiated phenotype of RMS cells (41). In particular, Rao et al (29) reported that overexpression of miR-1 in the RMS cell line, RD, results in muscle gene expression and cell cycle arrest, whereas miR-133a decreases the expression of muscle markers. This is consistent with the distinct roles of miR-1 and miR-133a in normal muscle differentiation. However, miRNAs suppress cell growth in the RMS cell line, indicating that cell context is important to the fate of miRNA regulation.

A similar growth inhibitory effect has also been confirmed by forced expression of either miR-1 or miR-206 in the RMS cell line in vitro and in vivo $(42,43)$. The induction of miR-1/206 precursor led to decreased myogenic differentiation in cell migration and inhibition of tumorigenic potential. Furthermore, the results of mRNA profiling prior to and following miR-206 transfection in RD18 cells revealed that $>700$ genes were modulated, including c-Met (43). The downregulation of c-Met by miR-1/206 led to a significant inhibition of RMS development, suggesting that the targeting of c-Met is one of the underlying mechanisms responsible for RMS development.

The anti-tumor capacity of the ectopic expression of the miR-1/206 cluster in RMS was further verified by the observation that these miRNAs directly regulate the expression of CCND2, a cell cycle gene (44). Overexpression of miR-1/206 demonstrated a strong promyogenic effect in RMS cells and downregulated the protein and transcript levels of CCND2. Additionally, miR-1/206 significantly downregulated PAX3 protein expression in the eRMS cell line, JR1, however, 
demonstrated no effect on the protein levels of PAX3 in an aRMS cell line, Rh30 (44). This finding highlights, once more, the importance of cell context in determining the response to miRNA modulation.

\section{Non-muscle-specific miRNAs in rhabdomyosarcoma}

In addition to myomiRs, numerous non-myomiRs are implicated in the regulation of RMS development. The deregulation of miR-29 was reported in a small cohort of aRMS in which nuclear factor- $\kappa \mathrm{B}$ activation led to overexpression of Yin Yang 1, resulting in sustained downregulation of miR-29b2/miR-29c and inhibition of myogenesis (45). In addition, decreased expression of miR-29, as well as miR-1/206, stabilized the RMS phenotype by targeting PAX3 and CCND2 (44). These findings reiterate that the RMS state is maintained by the deregulation of multiple miRNAs and their target genes, supporting a tumor suppressor role for these miRNAs.

Another miRNA linked to RMS is miR-183, which acts as an onco-miR in several types of cancer, including RMS, synovial sarcoma and colon cancer (46). Knocking down of miR-183 by anti-miR-183 treatment in tumor cells reduced cell migration in vitro and stimulated the expression of the tumor suppressor gene phosphatase and tensin homolog (PTEN), which in turn, promoted early growth response 1 (EGR1) expression, thus reinforcing the repression of cell migration (46). These results demonstrated that miR-183 has an oncogenic role through targeting two tumor suppressor genes, EGR1 and PTEN, and the deregulation of the fundamental miRNA regulatory network may be central to the development of several other tumor types.

Additionally, certain other non-myomiRs were described in the context of cell differentiation, migration or metastasis in RMS. Re-expression of miR-203 in RMS cells inhibited their proliferation and migration and promoted terminal myogenic differentiation by directly targeting p63 (47). miR-9a is another miRNA capable of inhibiting cell migration, which was found to directly target E-cadherin and was expressed in higher levels in aRMS than in eRMS, correlating with their metastatic potentials (48). In addition, in the two cultured cells and tumor implants, the growth of RMS was significantly arrested by miR-450b-5p, which was strictly regulated by TGF- $\beta 1$ (49).

Taken together, these data demonstrate the critical role of miRNAs in modulating target genes involved in one or more cellular function/process and the complexity of miRNA regulation and function in RMS development. In addition, these studies have revealed that small gene expression alterations, even if only occurring in one miRNA, may be affect the balance between pathological and physiological cell fate programs.

\section{8. miRNAs as novel therapeutic targets in rhabdomyosar- coma}

The widespread and crucial roles of miRNAs in RMS development and progression raise interesting prospects for exploiting miRNAs as novel therapeutic targets in RMS.

In this regard, various approaches that upregulate or downregulate miRNAs have been employed to target miRNAs in RMS, and demonstrate significant efficacy in the treatment of RMS development following intravenous delivery in vivo (50).
In particular, two pre-clinical studies demonstrated that ectopic expression of miR-206 by lentiviral vectors leads to cell cycle arrest and myogenic differentiation of RMS cells, preventing xenograft growth in vivo by inhibiting the expression of oncogenic c-Met $(42,43)$. In addition, knockdown of miR-183, an onco-miR in several types of cancer, by antisense-based miRNA antagonists led to significant decreases in tumor migration through directly promoting the expression of EGR1, a regulator of cell migration (46).

Although the upregulation or downregulation of selected miRNAs is a possible strategy for targeted therapy in RMS, it must be noted that there remain several challenges regarding miRNA-based therapy. Viral vectors, though efficient in the overexpression of miRNA genes, are limited in their clinical application by immunogenicity and non-specificity. Non-viral cationic liposomes are attractive for mediating miRNA transfer, however, their low efficiency in cell transfection also limit their development. Certain types of nanoparticles have been proposed to efficiently deliver miRNAs or anti-miRNAs to target tumor sites (51), implying that they are alternative tools for introducing miRNAs for the treatment of RMS. However, additional preclinical data are required to demonstrate their suitability for the clinic and efficacy in the application of miRNA therapy. Their relevance and mode of action require further investigation in genetic models of RMS that more accurately recapitulate the onset and progression of aRMS and eRMS tumors.

\section{Conclusion}

miRNAs have emerged as critical regulators in skeletal muscle development, regeneration and function. They are also found to be dysregulated in skeletal muscle-associated diseases, including RMS. Thus, miRNAs are promising biomarkers and candidates for potential therapeutic intervention, and provide an avenue to further dissect the mechanisms that may contribute to genetic and acquired muscle disorders or other associated diseases. Future studies are required to focus on the identification of miRNAs involved in skeletal muscle development and on advancing novel therapies that are able to modulate miRNA activity to treat muscle-associated diseases.

\section{Acknowledgements}

This study was supported by the Project Funded by the Priority Academic Program Development of Jiangsu Higher Education Institutions (PAPD), Jiangsu Co-innovation Center for Prevention and Control of Important Animal Infectious Diseases and Zoonoses and the National Natural Science Foundation of China (grant nos. 31101683 and 31272405).

\section{References}

1. Fong AP and Tapscott SJ: Skeletal muscle programming and re-programming. Curr Opin Genet Dev 23: 568-573, 2013.

2. Arndt CA, Rose PS, Folpe AL and Laack NN: Common musculoskeletal tumors of childhood and adolescence. Mayo Clin Proc 87: 475-487, 2012.

3. Perez EA, Kassira N, Cheung MC, Koniaris LG, Neville HL and Sola JE: Rhabdomyosarcoma in children: a SEER population based study. J Surg Res 170: e243-e251, 2011.

4. Charytonowicz E, Cordon-Cardo C, Matushansky I and Ziman M: Alveolar rhabdomyosarcoma: is the cell of origin a mesenchymal stem cell? Cancer Lett 279: 126-136, 2009. 
5. Hatley ME, Tang W, Garcia MR, et al: A mouse model of rhabdomyosarcoma originating from the adipocyte lineage. Cancer Cell 22: 536-546, 2012.

6. Barr FG: Molecular genetics and pathogenesis of rhabdomyosarcoma. J Pediatr Hematol Oncol 19: 483-491, 1997.

7. Novák J, Vinklárek J, Bienertová-Vašků J and Slabý O MicroRNAs involved in skeletal muscle development and their roles in rhabdomyosarcoma pathogenesis. Pediatr Blood Cancer 60: 1739-1746, 2013.

8. Bartel DP: MicroRNAs: genomics, biogenesis, mechanism and function. Cell 116: 281-297, 2004

9. Calin GA and Croce CM: MicroRNA signatures in human cancers. Nat Rev Cancer 6: 857-866, 2006.

10. Wang F, Fu XD, Zhou Y and Zhang Y: Down-regulation of the cyclin E1 oncogene expression by microRNA-16-1 induces cell cycle arrest in human cancer cells. BMB Rep 42 : 725-730, 2009.

11. Wang F, Niu G, Chen X and Cao F: Molecular imaging of microRNAs. Eur J Nucl Med Mol Imaging 38: 1572-1579, 2011.

12. Wang F, Song X, Li X, et al: Noninvasive visualization of microRNA-16 in the chemoresistance of gastric cancer using a dual reporter gene imaging system. PLoS One 8: e61792, 2013.

13. Ciarapica R, Russo G, Verginelli F, et al: Deregulated expression of miR-26a and Ezh2 in rhabdomyosarcoma. Cell Cycle 8: 172-175, 2009

14. Davicioni E, Anderson JR, Buckley JD, Meyer WH and Triche TJ: Gene expression profiling for survival prediction in pediatric rhabdomyosarcomas: a report from the children's oncology group. J Clin Oncol 28: 1240-1246, 2010.

15. Gougelet A, Perez J, Pissaloux D, et al: miRNA profiling: How to bypass the current difficulties in the diagnosis and treatment of sarcomas. Sarcoma 2011: 460650, 2011.

16. Bentzinger CF, Wang YX and Rudnicki MA: Building muscle: molecular regulation of myogenesis. Cold Spring Harb Perspect Biol 4: a008342, 2012.

17. Buckingham M: Skeletal muscle formation in vertebrates. Curr Opin Genet Dev 11: 440-448, 2001

18. Berkes CA and Tapscott SJ: MyoD and the transcriptional control of myogenesis. Semin Cell Dev Biol 16: 585-595, 2005.

19. Stockdale FE: Mechanisms of formation of muscle fiber types. Cell Struct Funct 22: 37-43, 1997.

20. Lu L, Zhou L, Chen EZ, et al: A novel YY1-miR-1 regulatory circuit in skeletal myogenesis revealed by genome-wide prediction of YY1-miRNA network. PLoS One 7: e27596, 2012.

21. Chen JF, Mandel EM, Thomson JM, et al: The role of microRNA-1 and microRNA-133 in skeletal muscle proliferation and differentiation. Nat Genet 38: 228-233, 2006.

22. Kim HK, Lee YS, Sivaprasad U, Malhotra A and Dutta A: Muscle-specific microRNA miR-206 promotes muscle differentiation. J Cell Biol 174: 677-687, 2006.

23. Dey BK, Gagan J and Dutta A: miR-206 and -486 induce myoblast differentiation by downregulating Pax7. Mol Cell Biol 31: 203-214, 2011.

24. Huang MB, Xu H, Xie SJ, Zhou $\mathrm{H}$ and Qu LH: Insulin-like growth factor-1 receptor is regulated by microRNA-133 during skeletal myogenesis. PLoS One 6: e29173, 2011.

25. McCarthy JJ: MicroRNA-206: the skeletal muscle-specific myomiR. Biochim Biophys Acta 1779: 682-691, 2008.

26. Zhao Y, Samal E and Srivastava D: Serum response factor regulates a muscle-specific microRNA that targets Hand2 during cardiogenesis. Nature 436: 214-220, 2005.

27. Sweetman D, Goljanek K, Rathjen T, et al: Specific requirements of MRFs for the expression of muscle specific microRNAs, miR-1, miR-206 and miR-133. Dev Biol 321: 491-499, 2008.

28. Zhao Y, Ransom JF, Li A, et al: Dysregulation of cardiogenesis, cardiac conduction and cell cycle in mice lacking miRNA-1-2. Cell 129: 303-317, 2007.
29. Rao PK, Missiaglia E, Shields L, et al: Distinct roles for miR-1 and miR-133a in the proliferation and differentiation of rhabdomyosarcoma cells. FASEB J 24: 3427-3437, 2010

30. Anderson C, Catoe H and Werner R: MIR-206 regulates connexin43 expression during skeletal muscle development. Nucleic Acids Res 34: 5863-5871, 2006.

31. Chen JF, Tao Y, Li J, et al: microRNA-1 and microRNA-206 regulate skeletal muscle satellite cell proliferation and differentiation by repressing Pax7. J Cell Biol 190: 867-879, 2010.

32. Rosenberg MI, Georges SA, Asawachaicharn A, Analau E and Tapscott SJ: MyoD inhibits Fstll and Utrn expression by inducing transcription of miR-206. J Cell Biol 175: 77-85, 2006.

33. Crist CG, Montarras D, Pallafacchina G, et al: Muscle stem cell behavior is modified by microRNA-27 regulation of Pax3 expression. Proc Natl Acad Sci USA 106: 13383-13387, 2009.

34. Wong CF and Tellam RL: MicroRNA-26a targets the histone methyltransferase Enhancer of Zeste homolog 2 during myogenesis. J Biol Chem 283: 9836-9843, 2008.

35. Feng Y, Cao JH, Li XY and Zhao SH: Inhibition of miR-214 expression represses proliferation and differentiation of $\mathrm{C} 2 \mathrm{C} 12$ myoblasts. Cell Biochem Funct 29: 378-383, 2011.

36. Liu J,Luo XJ, Xiong AW, et al: MicroRNA-214 promotes myogenic differentiation by facilitating exit from mitosis via down-regulation of proto-oncogene N-ras. J Biol Chem 285: 26599-26607, 2010.

37. Naguibneva I, Ameyar-Zazoua M, Polesskaya A, et al: The microRNA miR-181 targets the homeobox protein Hox-A11 during mammalian myoblast differentiation. Nat Cell Biol 8: 278-284, 2006.

38. Naguibneva I, Polesskaya A, Ameyar-Zazoua M, et al: Micro-RNAs and muscle differentiation. J Soc Biol 201: 367-376, 2007 (in French).

39. Crippa S, Cassano M, Messina G, et al: miR669a and miR669q prevent skeletal muscle differentiation in postnatal cardiac progenitors. J Cell Biol 193: 1197-1212, 2011.

40. Slater O and Shipley J: Clinical relevance of molecular genetics to paediatric sarcomas. J Clin Pathol 60: 1187-1194, 2007.

41. Chen JF, Callis TE and Wang DZ: microRNAs and muscle disorders. J Cell Sci 122 (Pt 1): 13-20, 2009.

42. Taulli R, Bersani F, Foglizzo V, et al: The muscle-specific microRNA miR-206 blocks human rhabdomyosarcoma growth in xenotransplanted mice by promoting myogenic differentiation. J Clin Invest 119: 2366-2378, 2009.

43. Yan D, Dong Xda E, Chen X, et al: MicroRNA-1/206 targets c-Met and inhibits rhabdomyosarcoma development. J Biol Chem 284: 29596-29604, 2009.

44. Li L, Sarver AL, Alamgir S and Subramanian S: Downregulation of microRNAs miR-1,-206 and -29 stabilizes PAX3 and CCND2 expression in rhabdomyosarcoma. Lab Invest 92: 571-583, 2012.

45. Wang H, Garzon R, Sun H, et al: NF-kappaB-YY1-miR-29 regulatory circuitry in skeletal myogenesis and rhabdomyosarcoma. Cancer Cell 14: 369-381, 2008.

46. Sarver AL,LiL and Subramanian S: MicroRNA miR-183 functions as an oncogene by targeting the transcription factor EGR1 and promoting tumor cell migration. Cancer Res 70: 9570-9580, 2010

47. Diao Y, Guo X, Jiang L, et al: miR-203, a tumor suppressor frequently down-regulated by promoter hypermethylation in rhabdomyosarcoma. J Biol Chem 289: 529-539, 2014.

48. Armeanu-Ebinger S, Herrmann D, Bonin M, et al: Differential expression of miRNAs in rhabdomyosarcoma and malignant rhabdoid tumor. Exp Cell Res 318: 2567-2577, 2012.

49. Sun MM, Li JF, Guo LL, et al: TGF-beta1 suppression of microRNA-450b-5p expression: a novel mechanism for blocking myogenic differentiation of rhabdomyosarcoma. Oncogene 33: 2075-2086, 2014.

50. Goljanek-Whysall K, Sweetman D and Münsterberg AE: microRNAs in skeletal muscle differentiation and disease. Clin Sci (Lond) 123: 611-625, 2012

51. Muthiah M, Park IK and Cho CS: Nanoparticle-mediated delivery of therapeutic genes: focus on miRNA therapeutics. Expert Opin Drug Deliv 10: 1259-1273, 2013. 